\title{
Evaluation of Developing Ratio Frequency Stealth Radar System
}

\author{
Zhao Yang \\ Ordnance Engineering College of PLA \\ Shijiazhuang,China \\ 935574660@qq.com \\ * Corresponding Author
}

Han ZHuangzhi

Ordnance Engineering College of PLA

Shijiazhuang,China

Zz_han@163.com

\author{
Shang CHaoxuan \\ Ordnance Engineering College of PLA \\ Shijiazhuang,China \\ shangchaoxuan@163.com
}

\begin{abstract}
Aimed at evaluating ratio frequency stealth of weapon system. This paper briefly introduces the the ratio frequency stealth technology development history of radar system, points out how radar system realize its ratio frequency stealth ability, and gives the index of evaluating ratio frequency stealth capability of radar system.Finally, introduces the limitation of the existing evaluation index.
\end{abstract}

Keywords-ratio frequency stealth; intercepting receiver; network radar; performance evaluation; intercept probability

\section{INTRODUCTION}

Stealth, aiming at reducing the target features, reduces the probability of its being recognized or estimated. Ratio frequency stealth of all kinds of weapons, including radar system, is to reduce the expose of the ratio frequency signal characteristics, make the enemy sensors(such as radar) fall into constantly processing, guessing and identifying the signal's information, thus let the enemy cannot find the target in time. Under the background of modern informational warfare, ratio frequency stealth weapons plays a more and more important role in real combat procedure .At home or abroad, the research of ratio frequency has been a hot topic.

\section{RESEARCH STATUS AT HOME AND ABROAD}

\section{A. the research status abroad}

Ratio frequency stealth is not new, foreign countries started the study of it in a earlier time. The predecessor of ratio frequency is low probability of intercept (LPI) technology. In 1941, during the second world war, in order to improve the weapon system's survival ability, Britain puts forward the RCS(radar cross-section) reduction technology. Ratio frequency stealth technology is a kind of electronic reconnaissance technology. On the Basis of the low probability of intercept technology, Ratio frequency stealth technology began to appear on the stage of history.

Ratio frequency stealth technology began to get rapid development in the 1970s. Different from the classic LPI technology, which compensates radar energy loss through signal processing so as to reduce the front intercept probability of electronic reconnaissance equipment, ratio frequency stealth technology compensates radar energy loss through all kinds of technologies, including radiation control, signal processing,and multiple sensor fusion, and finally reduces the system intercept probability of electronic reconnaissance equipment. Apparently, the latter is more compatible for modern informational warfare.

The first full experiment with low probability of intercept radar (LPIR) system was also born in this period. The result of this project finally confirmed, on the premise of guaranteeing the tracking accuracy of airborne radar, LPI technology can reduce their probabilities of being detected. This experiment verified the feasibility of ratio frequency stealth technology. From 1979 to 1980, the United States conducted the first airborne LPIR flight test, and successfully accomplished this experiment, which strengthened the power and confidence of researching ratio frequency stealth technology, from then on, further exploration in the field of ratio frequency stealth had been done.

\section{B. the domestic research status}

In the 21 st century, the domestic academia began to pay attention to the research of ratio frequency stealth technology . In 2004, the book Introduction to ratio frequency stealth firstly disclose some technical principle of ratio frequency stealth technology, which causes extensive concern in domestic radar field. Under the background of modern military competition, the electromagnetic environment becomes increasingly 
complex and radar countermeasures becomes more diverse.

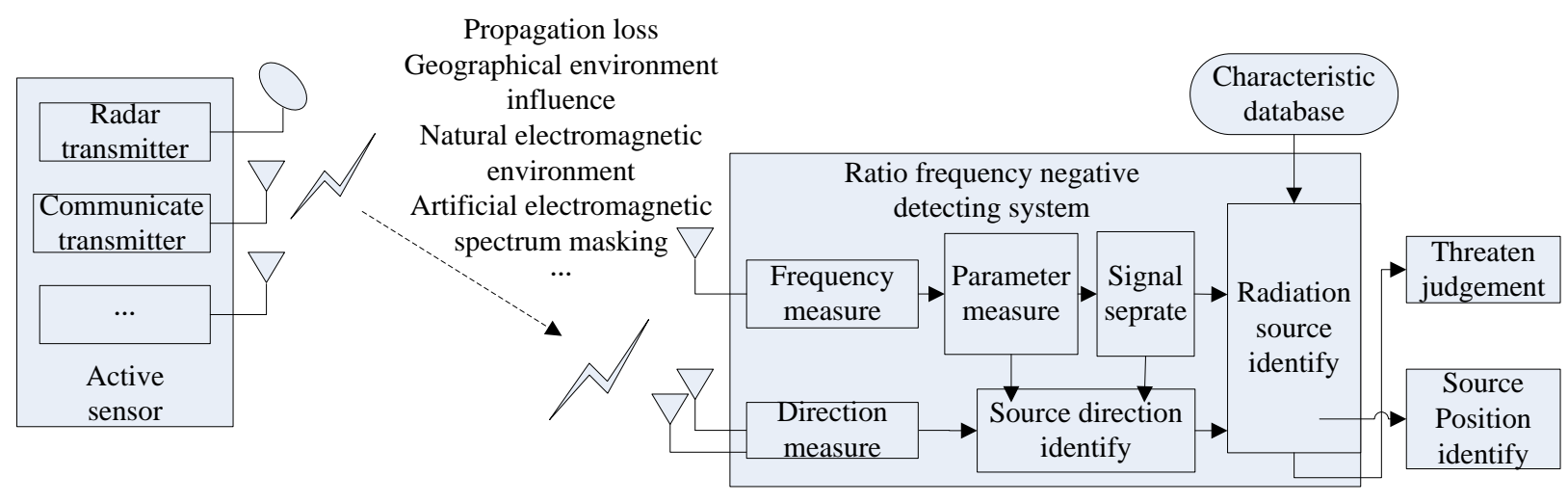

Figure 1. Scouted and identified process of ratio signal.

LPI radar technology, ratio frequency stealth technology, and other electronic reconnaissance technology become a research hot spot. At present, the electronic science and technology university, China institute of electronics, CLP, etc are carrying out many experiments on radar ratio frequency stealth technology, and have made great progress. With the deepening of the research, the lack of corresponding reasonable evaluation directly influence the energy and input of the ratio frequency stealth technology research, which is not conducive to the further development of the ratio frequency stealth technology.

\section{RATIO FREQUENCY STEALTH CAPABILITY}

\section{EVALUATION INDEX}

At present, the domestic and foreign radar research institutes have done a lot of fruitful work in the field of radar low intercept performance test evaluation[2-4], the evaluation of radar interception resistance indicators mainly include: intercept probability, intercept factor, intercept distance, etc. But if only one single low intercept index was used to evaluate radar ratio frequency stealth ability, the evaluation result would be only focused on one aspect of radar interception resistance assessment, which could not fully reflect the ratio frequency stealth performance of radar. To evaluate the capability of ratio frequency stealth, the signal sorting, radiating source recognition and threat judgment process of radar reconnaissance system should also be considered.

Currently there is no unified indicators towards the evaluation of ratio frequency stealth, through searching a large number of reference, synthesizing all aspects of the study, to evaluate the ratio frequency stealth performance of tracking radar, two basic indicators can be chosen:

- The front intercept probability: directly measure the intercepted probability of radar radiation signal.

- The probability of successfully sorting: reflect whether reconnaissance equipment could correctly sorting out the information of radar signal.

\section{A. the front intercept probability}

To intercept radar signals, Radar Warning Receiver (RWR) needs to intercept several aspects, first of all, space alignment is needed, which means the receiving antenna needs to focus on the radar signal; secondly, the power intercept is needed, which means the receiver sensitivity is greater than the received radar signal power; Thirdly, time intercept is needed, which means the receiver needs to receive a certain amount numbers of radar signal, and guarantee the possibility of subsequent processing; Finally frequency domain intercepted is also needed, which means that the receive ratio frequency signals must be in the frequency range of the receiver . To sum up, intercept probability can be subscribed through the following expression $^{[5][6] \text { : }}$

$$
\mathrm{P}_{\mathrm{d}}=\mathrm{k}_{\mathrm{a}} * \mathrm{P}_{\mathrm{p}} * \mathrm{P}_{\mathrm{t}} * \mathrm{P}_{\mathrm{f}}
$$

$P_{d}$ is the detection probability; $k_{a}$ is antenna ability to focus on radar transmit signal; $\mathrm{P}_{\mathrm{p}}$ is the probability of power intercept; $\mathrm{P}_{\mathrm{t}}$ is the probability of time intercept; and $P_{f}$ is the probability of frequency intercept.

\section{B. the probability of successfully separation}

After the front part of warning receiver intercepting radar signal, the characteristic parameters of different radar signals also need to be measured, to realize the separation of different radar signal. On the basis of that, feature extraction, target recognition and threat level judgment will be carried out. Through the study of all kinds of signal sorting methods, the implementation of radar signal sorting of current warning receiver mainly use the way of joint probability separation. Joint probability separation includes the separation of signal pulse repetition rate, carrier frequency, signal bandwidth, arrival angle of combination of multiple parameters ,etc. Compared with the single parameter separation, the joint parameter separation can greatly improved the final separation probability. To 
indicate the joint separation successful probability, the following parameter can be used ${ }^{[7-9]}$.

In a certain amount of time, the amount of signals received by RWR can be described as:

$$
\mathrm{N}=\mathrm{S} * \mathrm{~T}
$$

is the number of effective signals; is the sampling time; is the sampling rate.

then, the joint separation successful probability is:

$$
\mathrm{N} \quad \mathrm{P}=\frac{\max \left\{\mathrm{N}_{\mathrm{t}}, \mathrm{N}_{\mathrm{b}}, \mathrm{N}_{\mathrm{c}}, \mathrm{N}_{\mathrm{d}}\right\}}{\mathrm{T}}
$$

$\mathrm{N}_{\mathrm{t}}$ is the number sorted out from the analysis of pulse repeat interval; $\mathrm{N}_{\mathrm{b}}$ is the number sorted out from the analysis of bandwidth; $\mathrm{N}_{\mathrm{c}}$ is the number sorted out from the analysis of carrier frequency; $\mathrm{N}_{\mathrm{d}}$ is the number sorted out from the analysis of arriving angle.

\section{RADAR RATIO FREQUENCY STEALTH METHOD}

Based on the over mentioned evaluation indicators of the ration frequency stealth, and combining the already gotten experimental results, to realize ratio frequency stealth, radar system can use following methods.

\section{A. ratio frequency stealth of single radar}

\section{1) power control:}

Under each work mode of radar, the value of transmitted power need to be controlled. On the basis of ensuring the premise of target tracking, the transmission power needs to be reduced as lower as possible, which means that the conventional statement, maximum detection range of radar parameters, needs to be replaced by the statement of the minimum detectable distance. This measure ensure that the intercept receiver could only receive the minimum radar signal power, which will definitely play an important role in lowering the performance ability of intercept receiver.

\section{2) limit work time of radar transmitter:}

Ordinary tracking radar usually continue to illuminate the target to fulfill its mission of target tracking, the high target tracking accuracy will exposes its own position at the same time, which is extremely dangerous for their own survival. In the actual process of tracking, high-precision tracking can also be realized through separated illumination. The intermittent booting of transmitter, can make the intercept receiver cannot continuously receive radar signals, which will further influence the radar signal sorting and judging of intercept receiver.

\section{B. ratio frequency stealth of network radar}

Compared with the single radar, to realize ratio frequency stealth, network radar has more methods ${ }^{[10]}$. At present, the following points can be achieved on the technology layer.

1) intermittent radiation:

Single radar can also realize intermittent radiation,but it is easily to influence the tracking precision, even lost target, while the network radar has its unique advantage. Such as there are 10 different system of radar, working together to track the target at the same time, each single radar only need one over ten of the radiation time, compared with continuous tracking radar. Under this circumstance, network radar can also achieve continuous, high precision of target tracking, while at the same time, 10 different radar signal system will surely make it more difficult for intercept receiver to identify and judge the receiving signals.

2) Mismatch between generation and utilization gain:

Due to differences between the location and working system of the single radars in a netted radar system, the intercepted signals by the receiver may differ in amplitude, phase, frequency, gain and direction of arrival. Based on these differences, each single radar in the network system can be controlled to emit signals with different frequency, making the intercepted signals varying in amplitude and direction. In addition, the receiver tends to regard the singular signal as new component in signal assignment and accumulation. One can even use the bait to achieve the gain mismatch in order to enhance the security of the netted radar system.

\section{CONCLUSION}

Throughout the ratio frequency stealth development history, combined with background of information-based military struggle, it is not hard to see, in the field of ratio frequency stealth, there has a huge gap between our country and foreign countries.

The evaluating indicator of ratio frequency stealth is far from being completed. More work needs to be done in the future.

\section{REFERENCES}

[1] G. Eason, B. Noble, and I. N. Sneddon, "On certain integrals of Lipschitz-Hankel type involving products of Bessel functions," Phil. Trans. Roy. Soc. London, vol. A247, pp. 529-551, April 1955. (references)

[2] SANG Jianhua, CHEN Linyi. Air Vehicle RF Stealth Technology in Evolution[J]. Aeronautical Manufacturing Technology, 2011,23/24: 48-50.

[3] ZHU Yinchuan, Implication and Performance Metric Research of RF Stealth for Aircraft[J]. Telecommunication Engineering, 2013, 1: 6-11.

[4] WANG Lin, RF Stealth Technology and Development Thoughts on Aviation Platform[J]. Telecommunication Engineering, 2013, 8: 973-976.

[5] CAO Yunmei, WANG Ying, Overview on Development of Radar Stealth and Anti-Stealth Technologies of Radar, Modern Navigation, 2012, 3: 215-218.

[6] QIU Lei, He Xiwei, Wu Hongchao, A Main Sorting Algorithm for Airborne Radar Warning Receiver[J]. Shipboard Electronic Countermeasure, 2012, 1: 8-12.

[7] LI Jianwei, Threats to Radar Warning Receiver that Airborne Active Phased Array Radars Bring[J]. Radar \& ECM, 2014, 2: 42-46.

[8] LIU Biliu, Radar Signal Sorting Technology in the Complicated Electromagnetic Environment[J]. Aerospace Electronic Warfare, 2011, 5: 74-77.

[9] FENG Boyu, Computational Model of Radio Frequency Risk on Stealth Performance of Airborne Radar[J]. Systems Engineering and Electronics, 2013, 1: 11-15.

[10] LI Junzheng, Development on RF Stealth of Aircraft[J].Modern Navigation, 2012, 3: 33-36. 